\title{
Isolation and Identification of M. tuberculosis from Sheep Tissue Samples and Sero-Diagnosis Study in an Organized Sheep Farm
}

\author{
K. Arunmozhivarman ${ }^{1}$, R. Radhika ${ }^{1}$, P. Kannan ${ }^{2}$, V. Maroudam ${ }^{1}$, \\ K. Vijayalakshmi ${ }^{1}$, P. Valentina Claudet ${ }^{1}$ and G. Dhinakar Raj $^{1}{ }^{*}$
}

\author{
${ }^{1}$ Translational Research Platform for Veterinary Biologicals, Tamil Nadu Veterinary and \\ Animal Sciences University, Chennai -600 051, India \\ ${ }^{2}$ Department of Immunology, National Institute for Research in Tuberculosis, \\ Chennai-31, India \\ *Corresponding author
}

\section{A B S T R A C T}

A 2 years old female Madras Red sheep with the medical history of reduction in feed intake, poor weight gain and emaciation was found dead in an organised farm. The

Keywords

Sheep, $M$. tuberculosis, PCR, ELISA,

Seroprevalence

Article Info

Accepted:

20 December 2017

Available Online:

10 January 2018 sheep did not have any obvious respiratory symptoms. Edematous and caseous lesions were observed in mesenteric, bronchial, mediastinal and prescapular lymph nodes of the sheep during post mortem examination. Other internal organs were free of any specific lesions. The lymph node samples were decontaminated and cultured by inoculating into BACTEC Mycobacteria Growth Indicator Tube (MGIT) system and Lowenstein Jensen slants. The cultures turned positive and acid fast staining of the bacterial culturerevealed the presence of Mycobacteria. The bacteria was further confirmed as Mycobacterium tuberculosis by multiplex PCR and nucleotide sequencing. A Tuberculosis sero-diagnostic study was conducted for all the animals in the farm using commercially available ELISA kit to know the incidence of tuberculosis in the farm. Three sheep out of the total 205 sheep were positive for tuberculosis by ELISA with the estimated $1.5 \%$ positivity. This shows the active circulation of tuberculosis in sheep farm and there may be possibility of human to animal transmission and vice versa. The role of sheep in the epidemiology and transmission of tuberculosis needs further study.

\section{Introduction}

Tuberculosis (TB) is a chronic bacterial disease caused by Mycobacterium tuberculosis complex (MTC) leading to decreased productivity, economic losses and poses a significant threat to human health. Among
MTC organisms, the major agents are $M$. tuberculosis and $M$. bovis. The primary host for $M$. bovis is cattle and M. tuberculosis is human. However, occurrence of $M$. tuberculosis in animals and $M$. bovis infection in humans has been reported previously (Ocepek et al., 2005). 
In ovine, the occurrence of tuberculosis is very rare although there are few reports indicating the presence of $M$. bovis in sheep and goat (Kassa et al., 2012; Marianelli et al., 2010). This primarily occurs in areas with high intensity sheep population and when there exists close contact between infected cattle and sheep facilitating transmission between these species. India accounts for one fourth of the global TB burden (Central TB division, GOI, 2017).

Tuberculosis in animals is not well studied in India; the lack of nation-wide epidemiological studies makes the disease burden largely unknown (Neeraja et al., 2014a). Few studies have documented the prevalence of TB in animals in India (Parmer et al., 2014; Srivastava, 2008). Tuberculosis causes huge economic loss in farm animals and the production loss in infected animals will be 10 to 20 percent (Verma et al., 2004).

Tuberculosis is often unnoticed in animals and the infected animals continue to spread the disease to other susceptible animals and human by excreting the organisms through milk, faeces and respiratory droplets. Hence to control tuberculosis both animals and human has to be monitored for disease prevalence. In this study Mycobateria was isolated from a TB infected sheep and $M$. tuberculosis was identified by multiplex PCR and gene sequencing. Then all the sheep in the farm were screened for TB sero-positivity.

\section{Materials and Methods}

\section{Sample collection}

Post mortem examination was carried out on one Madras red sheep that had died in an organized farm. The mesenteric, pre-scapular, bronchial and mediastinal lymphnodes were edematous and caseous. Samples from these lymphnodes were collected in sterile PBS and transported to laboratory on ice for mycobacterial culture.

\section{Isolation Mycobacterium Sp. from tissue samples}

The samples were decontaminated and processed following the modified Petroff's method (Kent and Kubica, 1985). A portion of the decontaminated sediments were inoculated into Mycobacterial Growth Indicator Tubes $(\mathrm{MGIT})^{\mathrm{TM}}$ from Becton Dickinson (BD) and incubated in BACTEC MGIT 960 instrument for 49 days at $37^{\circ} \mathrm{C}$.

The remaining sediments were inoculated into one tube each of OADC-supplemented Middlebrook 7H10 agar and LowensteinJensen (LJ) medium with sodium pyruvate and glycerol and each tube was incubated for 8 weeks at $37^{\circ} \mathrm{C}$.

\section{Acid fast staining}

Heat fixed smears prepared from the sediment and MGIT cultures declared as positive by the BACTEC 960 and typical growths on Middlebrook $7 \mathrm{H} 10$ and LJ media were screened for presence of acid fast bacilli. The heat-fixed smears were stained for acid fast bacilli as per the standard protocol.

\section{Polymerase chain reaction confirmation and sequencing}

The DNA extraction from MGIT liquid culture and colonies on $7 \mathrm{H} 10$ agar/LJ media was performed according to the CTAB $-\mathrm{NaCl}$ method. These DNA samples were subjected to conventional polymerase chain reaction (PCR) with specific primers reported by Zumarrga et al., (1999) and Bakshi et al., (2005). Then amplified PCR products were sequenced to confirm the mycobacterium species. 
Sero prevalence study using ELISA

Sheep sera samples from study farm were screened for tuberculosis antibodies using the commercial ELISA kit (IDEXX), USA as per manufacturer's instruction.

\section{Result and Discussion}

The post mortem caseous, edematous lymphnode tissue samples collected from the tuberculosis-suspected sheep were subjected to acid fast staining, bacterial culture and PCR. Staining of tissue smear from sheep lymph node revealed that presence of rod shaped, acid fast bacilli indicating the presence of mycobacterium infection (Figure 1a).
Bacterial culture study is the gold standard for laboratory confirmation of TB. Hence the tissue samples were cultured in LJ medium resulting in colonies that were rough, granular and whitish initially and later on the colonies turned yellowish (Figure 1b).

DNA amplification by PCR provides a rapid and sensitive method for the detection of $M$. tuberculosis complex (MTC) from postmortem samples and cultures (Clarridge et al., 1993). DNA extracted from LJ medium culture were subjected to multiplex PCR method. PCR product was further analyzed by agarose gel electrophoresis. There was no band around $168 \mathrm{bp}$ which is $M$. bovis specific whereas $M$. tuberculosis specific band around $337 \mathrm{bp}$ was visualized (Figure 2).

Fig.1a Acid Fast bacilli in Ziehl-Neelsen staining; Fig.1b Characteristic Mycobacterium colonies on Lowenstein Jensen medium

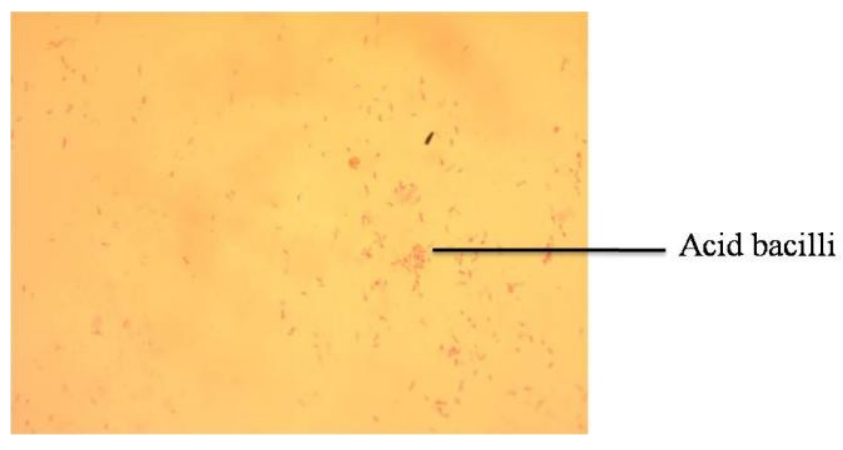

1a

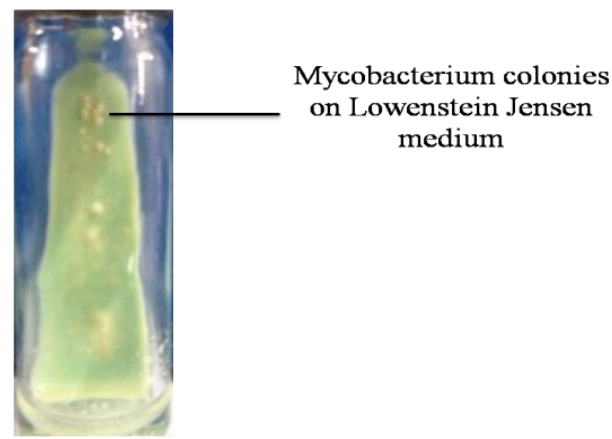

$1 b$

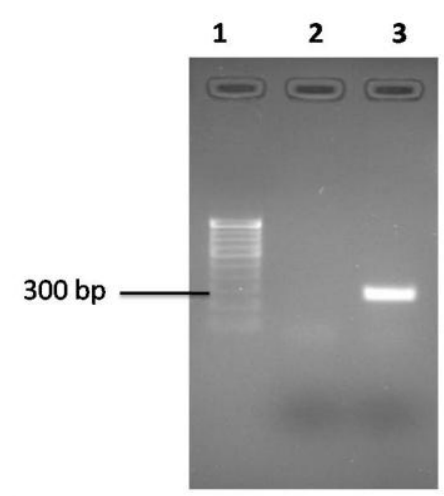

Figure 2 - Multiplex PCR; Lane 1 - 100 bp marker

Lane 2 - Negative control; Lane 3 - Sample 
The PCR product was subjected to gene sequencing and confirmed as $M$. Tuberculosis.

Further, the circulation TB in the sheep farm was identified by using ELISA to estimate sero-prevalence. Generally humans are the maintenance hosts for $M$. tuberculsois. The sheep is considered to be the spill-over hosts for $M$. bovis, can maintain the organism only when its population density is high and is generally considered very rare in small ruminants (Tschopp et al., 2011).

However, presence of MTB in sheep indicates a possible transmission of infection from human to animal. In this study out of 205 sheep 3 were sero-positives and indicates $1.5 \%$ sero-prevalence of TB was observed in study population. Lack of a robust animal TB surveillance system and vaccine use in animals aids in the transmission of TB between animals and from animals to human or vice versa. Thus there is an urgent and unmet need for implementation of animal TB control programs in developing countries through extensive surveillance. The license for the use of BCG vaccine in animals also warrants further studies.

\section{References}

Bakshi, C. S., D. H. Shah, R. Verma, R. K. Singh and M. Malik, 2005. Rapid differentiation of Mycobacterium bovis and Mycobacterium tuberculosis based on a $12.7-\mathrm{kb}$ fragment by a single tube multiplex-PCR. Vet Microbiol., 109: 211-6.

Clarridge, J. E., R. M. Shawaz, T. M. Shinnick and B. B. Plikaytis, 1993. Large scale use of PCR for detection of Mycobacterium tuberculosis in routine mycobacteriology laboratory. J. Clin. Microbiol., 31: 2049-2056.

Gezahegne MamoKassa, Fekadu Abebe,
Yalelet Worku, Mengistu Legesse, Girmay Medhin, Gunnar Bjune and Gobena Ameni, 2012. Tuberculosis in Goats and Sheep in Afar Pastoral Region of Ethiopia and Isolation of Mycobacterium tuberculosis from Goat. Vet Med Int., 2012: 869146.

Kent. P. T., and Kubica G. P., 1985. Public Health Mycobacteriology a guide for the level III laboratory. Centre for Disease Control Manual, Pp. 21-44.

Marianelli, C., N. Cifani and M. T. Capucchio, 2010. A case of generalized bovine tuberculosis in a sheep. Journal of Veterinary Diagnostic Investigation, 22(3): 445448.

Neeraja, D., B. M. Veeregowda, M. S. Rani, D. Rathnamma, R. Bhaskaran, L. Gowda, S. H. Somshekhar, M. Saminathan, K. Dhama and S. Chakraborty, 2014. Comparison of Single Intradermal Test, Gamma Interferon Assay and Indirect ELISA for the Diagnosis of Tuberculosis in a Dairy Farm. Asian Journal of Animal and Veterinary Advances, 9: 593-598.

Ocepek, M., M. Pate, M. Zolnir-Dovc and M. Poljak, 2005. Transmission of Mycobacterium tuberculosis from human to cattle. J. Clin. Microbiol., 43: 3555-3557.

Parmar, B. C., M. N. Brahmbhatt, J. B. Nayak, A. J. Dhami and Y. A. Chatur, 2014. Prevalence of tuberculosis in men and animals: Confirmation by cultural examinations, tuberculin tests and PCR technique. Journal of Foodborne and Zoonotic Diseases, 2: 36-44.

Srivastava, K., D. S. Chauhan, P. Gupta, H. B. Singh, V. D. Sharma and V. S. Yadav, 2008. Isolation of Mycobacterium bovis and M. tuberculosis from cattle of some farms in north India - possible relevance in 
human health. Indian J Med Res., 128: 26-31.

Tschopp, R., K. Bobosha, A. Aseffa, E. Schelling, M. Habtamu, R. Iwnetu, E. Hailu, R. Firdessa, J. Hussein, D. Young and J. Zinsstag, 2011. Bovine tuberculosis at a cattle-small ruminant-human interface in Meskan, Gurage region, Central Ethiopia. BMC Infectious Diseases, 11:318.

Verma, S., N. K. Mahajan and G. Malik,
2004. An epidemiological study on bovine H.S. in Haryana, Indian J Anim Res., 38(1): 14-19.

Zumarraga, M., F. Bigi, A. Alito, M. I. Romano and A. Cataldi, 1999. A 12.7 $\mathrm{kb}$ fragment of the Mycobacterium tuberculosis genome is not present in Mycobacterium bovis. Microbiology, 145: 893-897.

\section{How to cite this article:}

Arunmozhivarman, K., R. Radhika, P. Kannan, V. Maroudam, K. Vijayalakshmi, P. Valentina Claudet and Dhinakar Raj, G. 2018. Isolation and Identification of M. tuberculosis from Sheep Tissue Samples and Sero-Diagnosis Study in an Organized Sheep Farm. Int.J.Curr.Microbiol.App.Sci. 7(01): 2740-2744. doi: https://doi.org/10.20546/ijcmas.2018.701.328 